\title{
GEOQUÍMICA DE ISÓTOPOS ESTÁVEIS DE CARBONO DE ROCHAS CARBONOSAS DO QUADRILÁTERO FERRIIFERO, MINAS GERAIS
}

\author{
ROBERTO VENTURA SANTOS*, SELMA FERNANDES**, MESSIAS \\ GILMAR DE MENEZES** \& CLAUDINEI GOUVEIA DE OLIVEIRA**
}

\begin{abstract}
CARBON STABLE ISOTOPES OF CARBONACEOUS ROCKS FROM THE IRON QUADRANGLE, MINAS GERAIS STATE. We present and discuss the petrography and carbon stable isotope data on carbonaceous materials from tertiary and precambrian rocks from the Iron Quadrangle. Carbonaceous material in the Tertiary sediments presents a narrow range of $\delta^{13} \mathrm{C}(28.3$ to $-25 \%)$ and larger concentrations of total organic carbon if compared to the precambian rocks $(-29.8$ to $-5.9 \%)$. This narrow range of $\delta^{13} \mathrm{C}$ indicates that the carbon isotopic compositions of these rocks have not been significantly affected by diagenetic and post-diagenetic processes. The precambrian samples have, in general, less than $1 \mathrm{wt} \%$ total carbon. The wide range of $\delta^{13} \mathrm{C}$ of these rocks was related to the original isotopic composition of the rocks, as well as to sedimentary and metamorphic processes. We suggest that these processes led to volatilization of carbon compounds, such as $\mathrm{CO}, \mathrm{CO}_{2}$ and $\mathrm{CH}_{4}$. Even though we have not characterized the conditions of volatilization, we further suggest that they may be related to the presence of volatiles during the metamorphism. The carbonaceous material shows a broad peak in the same region of the main peak of graphite, indicating a low degree of crystallization.
\end{abstract}

Keywords: Carbon isotopes, graphite schists, Quadrilátero Ferrifero. Brazil

\begin{abstract}
RESUMO São apresentados e discutidos dados petrográficos e de isótopos de carbono em material carbonoso de sedimentos terciários e filhos precambianos do Quadrilátero Ferrifero. As amostras terciárias apresentam pequena variação de $\delta^{13} \mathrm{C}(-28.3$ a $-25.7 \%$ ) e teores significativamente mais elevados de carbono orgânico total se comparada às amostras precambianas $\left(-29.8 \mathrm{a}-5.9 \%\right.$ ). A pequena variação de $\delta^{13} \mathrm{C}$ dessas amostras indica que elas foram pouco afetadas por processos diagenéticos e pós-diagenéticos. As amostras precambianas possuem, em geral, concentração de carbono orgânico total abaixo de $1 \%$ por peso. A maior variação de $\delta^{13} \mathrm{C}$ se comparada às amostras terciárias foi relacionada à composição isotópica original das rochas e a processos sedimentares e matamórficos. Esses processos teriam sido responsáveis pela quebra dos compostos orgânicos originalmente presentes nas rochas e volatilização de compostos de carbono na forma $\mathrm{CO}$, $\mathrm{CO}_{2}$ e $\mathrm{CH}_{4}$. Muito embora não se saibam as condições em que essa volatilização tenha ocorrido, sugere-se que esse processo tenha sido uma possível fonte de voláteis (e.g. $\mathrm{CO}_{2}$ e $\left.\mathrm{CH}_{4}\right)$ durante o metamorfismo. Através de difratometria de raios-X, mostrou-se que o material carbonoso apresenta um baixo grau de cristalinidade, muito embora apresente um largo pico na região do pico principal da grafita.
\end{abstract}

Palavras-chave: Isótopos de carbono, grafita xistos, Quadrilátero Ferrifero, Brasil

INTRODUÇÃO O termo carbonoso refere-se a compostos orgânicos ou inorgânicos de carbono dispersos tanto em rochas metamórficas quanto não metamórficas. Este tipo de material tem sido descrito em rochas de idade arqueana a recente, constituindo um forte indício da existência de um reservatório de carbono orgânico desde o Arqueano. A composição isotópica de carbonates marinhos durante o período geológico mostra que houve pequenas variações no balanço entre carbono inorgânico (de carbonates) e carbono orgânico (Broecker 1970; Veizer et al 1980; Dês Marais et al 1992). Distúrbio desse balanço é verificado, no entanto, durante períodos glaciais, tais como no final do neoproterozóico (Margaritz et al 1986; Derry et al 1992; Kaufman et al 1993), ou durante períodos de extinção em massa, tais como no Permiano e no Cretáceo. Durante esses períodos, observa-se uma diminuição dos valores de $\delta^{13} \mathrm{C}$ de carbonates marinhos, que tem sido interpretada como uma diminuição do reservatório de carbono orgânico relativamente ao de carbono inorgânico.

Alguns estudos têm procurado mostrar a importância dos filitos carbonosos no processo de concentração de depósitos auríferos (Springer 1985; Volkov \& Fomina 1974; Guha et al 1984). O material carbonoso poderia atuar de várias formas, tais como desestabilizando por redução e adsorção os complexos auríferos. A adsorção de metais por compostos de carbono tem sido descrita em bacias euxínicas do Mar Negro (Volkov \& Fomina 1974). A importância do material carbonoso na concentração de ouro em depósitos precambrianos foi estudada por Springer (1985), que sugere que a transformação do material carbonoso em semi-grafita poderia ter uma atuação semelhante ao do carvão ativado utilizado no processo industrial de concentração de ouro. Além disso, há autores que sugerem a importância da condutibilidade de sulfetos na desestabilização de complexos de ouro (Starling et al 1989). Segundo esses autores, há uma tendência para o ouro precipitar-se em defeitos, faces, bordas e fraturas de cristais de pirita, que são consideradas zonas de máxima condutibilidade e polarização. Dessa forma, o estudo de rochas carbonosas torna-se importante para se avaliar sua atuação como agente redutor e adsorvente, bem como na distribuição de cargas elétricas em depósitos de ouro.

O Quadrilátero Ferrifero constitui uma das regiões mais bem estudadas do Brasil e com maior potencial metalògenético para ouro e ferro. Rochas carbonosas distribuem-se por quase toda a coluna estratigráfíca do Quadrilátero Ferrifero, sendo encontradas desde o Arqueano ao Recente. No Arqueano essas rochas estão associadas a importantes depósitos de ouro, tais como as minas de Morro Velho, São Bento, Cuiabá e Raposos. Material carbonoso também é encontrado junto aos conglomerados da Formação Moeda e filitos da Formação Barreiro, do Paleoproterozóico, e na bacia terciária do Gandarela. Os sedimentos da Bacia do Gandarela são tipicamente continentais e apresentam um vasta assembléia palinológica descrita por Lima \& SalardCheboldaeff (1981) e Pinto \& Pares-Regali (1991).

\footnotetext{
* Universidade de Brasília, Instituto de Geociências, 70910-900 Brasília - DF

** Universidade Federal de Ouro Preto, Departamento de Geologia, 35400-000 Ouro Preto - MG
} 
Os filhos carbonosos do Quadrilátero Ferrífero são tidos como de provável origem orgânica. Entretanto, poucos são os estudos que abordaram o ambiente de sedimentação e as condições metamórficas. Este trabalho tem por objetivo apresentar e discutir dados de isótopos estáveis de carbono em filhos carbonosos do Quadrilátero Ferrifero. São apresentados também dados petrográficos e de difração de raios-X, a partir dos quais tenta-se avaliar o grau de cristalinidade do material carbonoso.

ASPECTOS GEOLÓGICOS REGIONAIS A região Quadrilátero Ferrifero pode ser subdividida em seis grandes unidades precambrinas, conforme apresentadas nas Figuras 1 e 2. Enquanto que o Embasamento Cristalino é constituído

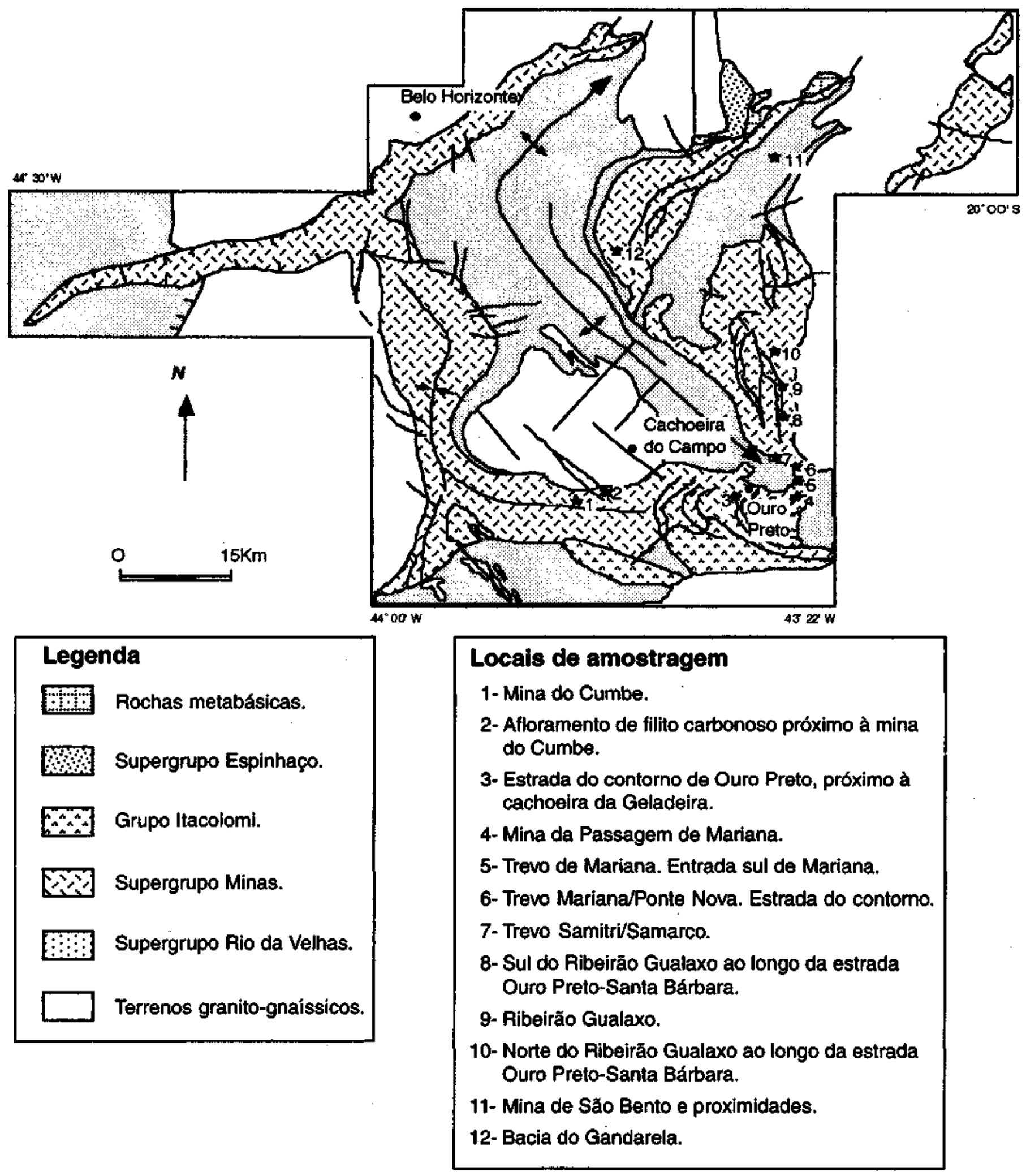

Figura 1 - Mapa geológico simplificado do Quadrilátero Ferrifero com a localização das amostras deste estudo Figure 1- Squematic geological map of the Iron Quadrangle showing the location of the samples of this study 

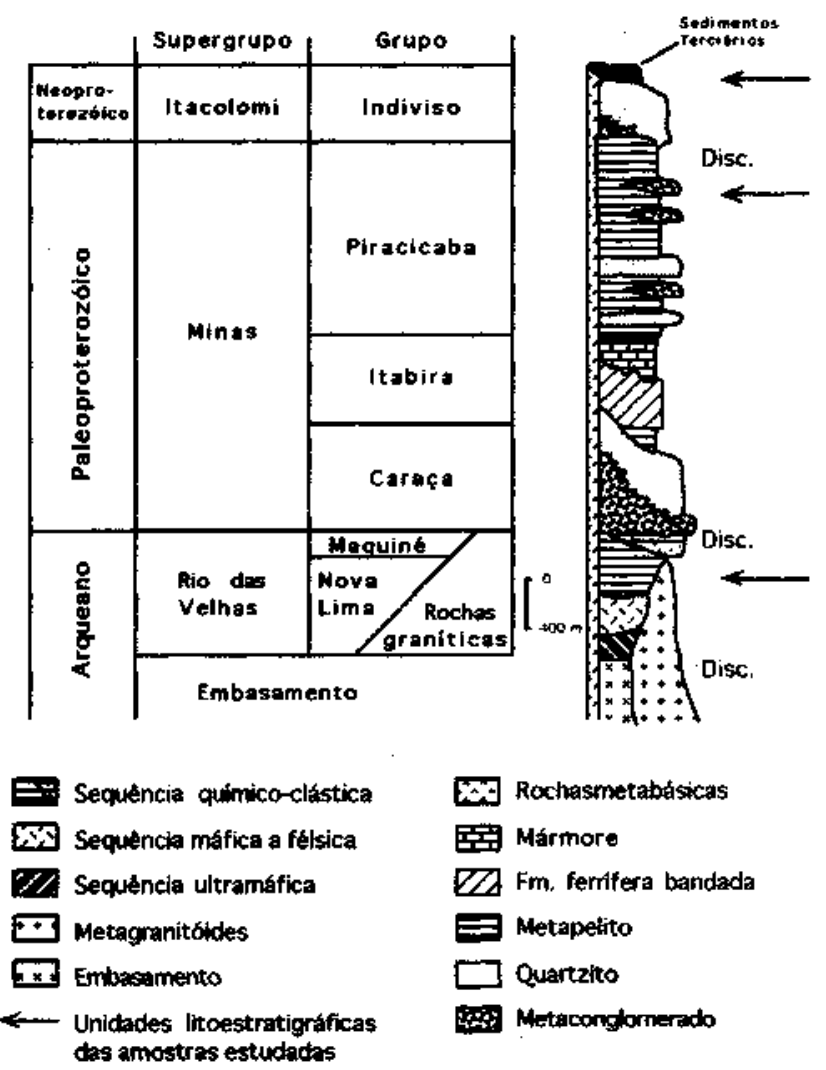

Figura 2 - Coluna estratigráfica simplificada do Quadrilátero Ferrifero mostrando o posicionamento estratigráfico das amostras deste estudo (mofidicado de Chemale et al. 1994)

Figure 2 - Stratigraphic column of the Iron Quadrangle showing the stratigraphic Iposition of the samples of this study (modified from Chemale et al. 1994)

predominantemente por gnaisses, as demais unidades são compostas predominantemente por rochas de origem vulcanogênica e sedimentar.

O Supergrupo Rio da Velhas representa uma seqüência vulcano sedimentar do tipo greenstone belt, sendo constituído por rochas metavulcânicas ácidas a ultramáficas e metassedimentos psamo-pelíticos e químicos.

O Supergrupo Minas é subdivido em três unidades denominadas Caraça, Itabira e Piracicaba (Ladeira 1980). Dentre estas, os Grupos Caraça e Piracicaba são predominantemente elásticas e constituídos por quartzitos e quartzito sericíticos, filitos arenosos, além de conglomerados que ocorrem na Formação Moeda. No Grupo Itabira dominam sedimentos químicos, principalmente formações ferríferas e rochas carbonáticas. Filitos carbonosos ocorrem na Formação Barreiro (Grupo Piracicaba) e, em menor proporção, nos conglomerados da Formação Moeda (Grupo Caraça).

Sobrepostos discordantemente ao Supergrupo Minas ocorrem quartzitos, quartzitos conglomeráticos e lentes de conglomerado com seixos de itabirito do Grupo Itacolomi.

As unidades terciárias recobrem estratigraficamente as unidades precambrianas nas Quadrículas de Gandarela e Santa Rita Durão/Catas Altas, ocorrendo respectivamente nas bacias Gandarela e Fonseca (Gorceix 1884).

AMOSTRAS E METODOLOGIA Foram estudadas 17 amostras da região do Quadrilátero Ferrifero, com idades que variam do Arqueano ao Terciário. As amostras arqueanas são provenientes da mina de Ouro de São Bento, enquanto que as amostras Proterozóicas são provenientes da Formação Gandarela e do Grupo Piracicaba e Caraça. A amostras terciárias são provenientes da Bacia do Gandarela, que são descritas como sedimentos continentais. O mapa da Figura 1 apresenta a localização das amostras estudadas.

As análises de $\delta^{13} \mathrm{C}$ foram realizadas no CENA (Centro de Energia Nuclear na Agricultura), Piracicaba, SP, através de combustão do material carbonoso em atmosfera de oxigênio. $\mathrm{O} \mathrm{CO}$ liberado a partir da combustão foi posteriormente analisado para isótopos de carbono por espectrometria de massa. Detalhes sobre essa técnica podem ser encontrados em Hoefs (1980) e Faure (1986). Antes de serem analizadas as amostras foram atacadas com ácido clorídrico $(10 \%)$, visando eliminar carbonatos. Os dados de isótopos de carbono são apresentados relativamente ao padrão PDB e em valores por mil:

$$
\delta^{13} \mathrm{C}=[(\mathrm{Rs}-\mathrm{Ra}) / \mathrm{Rs}] \times 1000,
$$

onde Ra e Rs referem-se às razões $\delta^{13} \mathrm{C} /{ }^{12} \mathrm{C}$ da amostra e do padrão, respectivamente.

O grau de cristalinidade do material carbonoso foi estudado através de difratometria de raios-X, no laboratório de difração de raios-X da Universidade Federal de Ouro Preto. Como o pico principal da grafita e do material carbono são semelhantes ao pico principal do quartzo, as amostras ricas em quartzo foram tratadas com ácido fluorídrico concentrado antes de serem analisadas por difratometria de raios-X. Nas amostras analisadas, comparou-se a forma do pico principal do material carbonoso com grafita de alto grau de cristalinidade e mediu-se o espaçamento d002.

$\mathrm{O}$ teor total de carbono foi analisado no CENPESPETROBRÁS, Rio Janeiro, RJ, e no Departamento de Metalurgia da Universidade Federal de Ouro Preto, Ouro Preto, MG.

Petrografia Esse tópico tem por objetivo descrever a forma de ocorrência do material carbonoso e suas relações com fílossilicatos e carbonatos. $\mathrm{O}$ estudo petrográfíco foi realizado nas amostras da Mina de São Bento e em rochas do Grupo Piracicaba.

As rochas carbonosas da Mina de São Bento, MG, possuem cor negra a cinza escura e xistosidade bem desenvolvida, que grada para uma foliação milonítica. O material carbonoso ocorre principalmente na forma de filmes com espessura menor que 0.2 milímetros e em geral orientados paralelamente à foliação principal (Fotografia 1). A textura milonítica é evidenciada pela presença de "augens" com sombra de pressão, foliação anastomótica, foliação S-C e bandas de cisalhamento. A porcentagem de material carbonoso é em geral menor que 5\% da composição modal, muito embora a cor negra das amostras sugira valores mais elevados. Os filmes de material carbonoso associam-se em geral a carbonatos e filosilicatos. O carbonato é truncado pela foliação milonítica, ao longo da qual se concentram os filmes de material carbonoso (Fotografia 2). Observa-se ainda que em algumas amostras a presença de material carbonoso associado a carbonato depende do domínio de deformação, ou seja, da intensidade da deformação. Nessas amostras há um aumento da proporção de material carbonoso das zonas menos deformadas, onde predomina carbonato não orientado, para as zonas mais deformadas, onde a foliação milonítica se encontra bem desenvolvida e a presença de carbonato é desprezível.

As amostras da Formação Fecho do Funil apresentam menor grau de deformação, muito embora a diversidade de texturas do material carbonoso seja semelhante à encontrada 


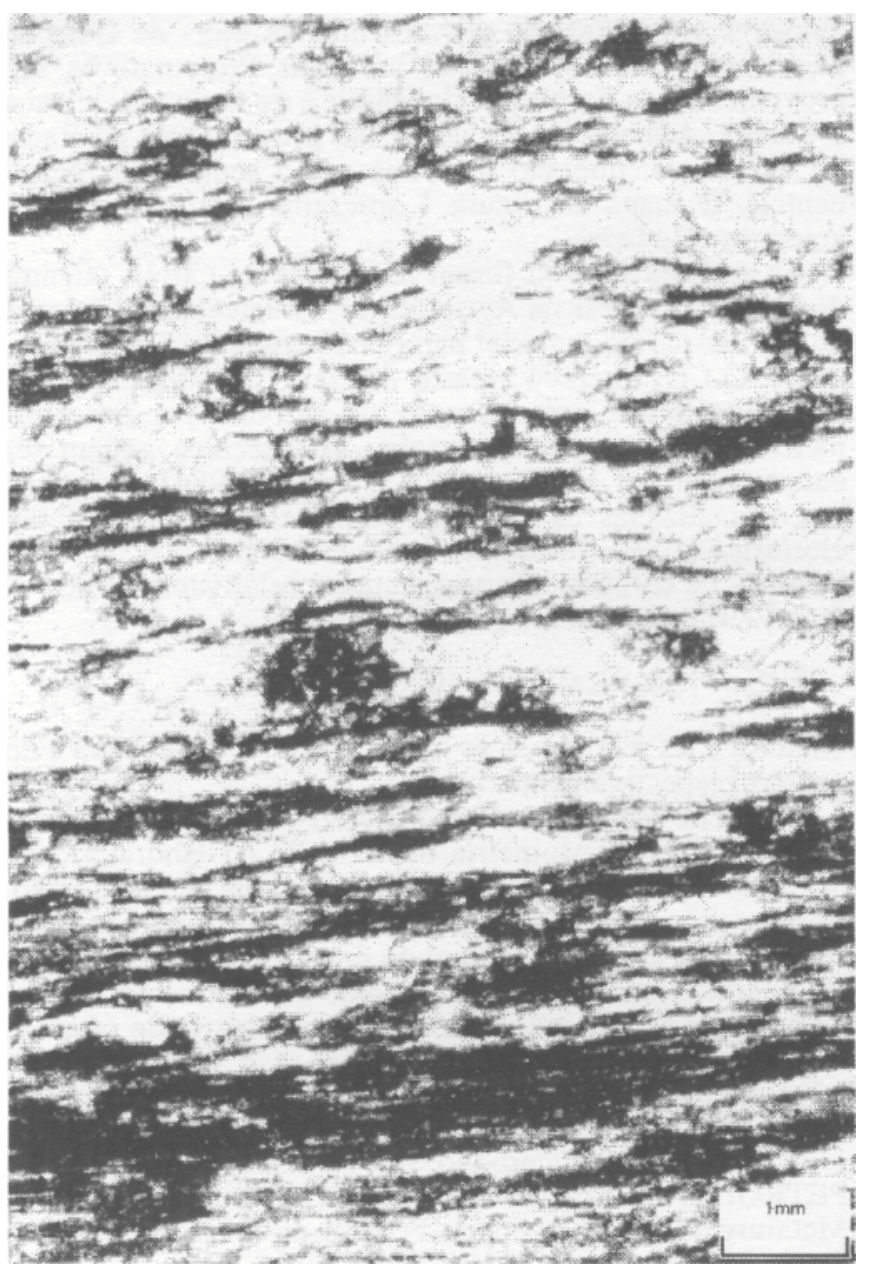

Fotografia 1 - Filito carbonoso a Mina de São Bento contendo quartzo, carbonato, filosilicatos e material carbonoso. Observar que o material carbonoso e os filosicatos ocorrem na forma de filmes orientados paralelos à foliação principal. Amostra SBM-T1. (N//) Photography 1 - Carbonaceous philite from the São Bento gold mine consisting of quartz, carbonate, philosilicates, and carbonaceous material. The carbonaceous material and the philosilicates occurs as films oriented parallel to the foliation. Samples SBM-T1. (Parallel nicols)

nas amostras acima. A presença de filmes de material carbonoso ocorre principalmente nas zonas mais deformadas, enquanto a presença de estruturas semi-esféricas são locais. Nas porções mais pelíticas é possível observar as relações entre o material carbonoso e clivagem de dissolução, onde o material carbonoso se orienta paralelamente à foliação principal e ao longo dos planos de xistosidade (Fotografia 3).

As amostras da Bacia do Gandarela possuem cor cinza escura e, ao contrário das anteriores, não apresentam evidências de deformação. São rochas laminadas, quebradiças e com fragmentos de restos vegetais (e.g. folhas, caules e raizes) (Duarte \& Mello 1980).

RESULTADOS Foram analisadas 17 amostras de rochas carbonosas para isótopos de carbono, sendo 18 do Quadrilátero Ferrifero, uma amostra de grafita da Mina Nacional de Grafite em Itapecirica, MG, e uma amostra de filito carbonoso do greenstone belt de Crixás, GO. Os dados encontram-se na tabela 1 e apresentados graficamente na Figura 3. Com exceção de uma amostra da Mina de Passa-

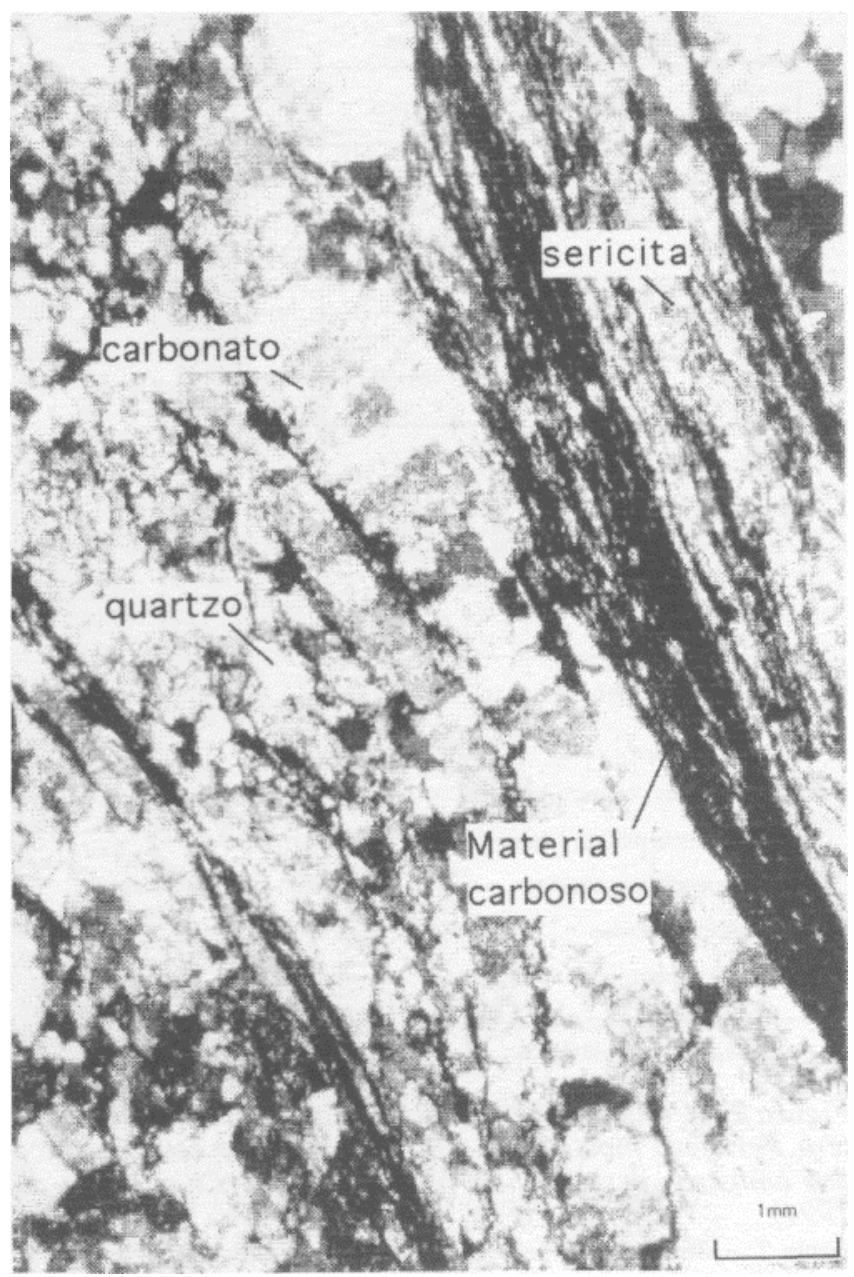

Fotografia 2 - Fotoinicrografia mostrando quartzo, carbonato, filossilicato e material carbonoso. Observar o truncamenio dos cristais de quartzo e carbonato pela foliação milonítica, bem como a disposição em filmes do material carbonoso e filossilicatos. Amostra SBM-T2. (NX) Photography 2- Phylite consisting of quartz, carbonate, phylosilicate, and carbonaceous material. Note the carbonate and quartz crystals disrupted by the mylonitic foliation, which is marked by films of carbonaceous material and philosilicates. (Sample SBM-T2). (Crossed nicols)

gem de Mariana, os valores de $\delta^{13} \mathrm{C}$ encontram-se dentro de espectro da variação de rochas carbonosas derivadas de material orgânico.

No gráfico da Figura 3, que compara o teor de carbono orgânico total versus os valores de $\delta^{13} \mathrm{C}$, observa-se uma separação entre as amostras de idade terciária (não metamórficas) e as precambrianas. De um modo geral, as rochas terciárias exibem valores de carbono orgânico total mais elevados e uma menor variação nos valores de $\delta^{13} \mathrm{C}$.

Utilizou-se da técnica de Difração de Raios X (DRX) para estudar o grau de cristalinidade da grafita através da medição do valor do espaçamento interplanar (d), reflexão 002 na posição 2Q ou d2-dl (Griffin, 1967; Buseck \& BoJun, 1985). Prepararam-se 45 amostras para análise de $\mathrm{DRX}$, que foram finamente moidas e submetidas à análise de pó numa ampla varredura de 20 entre 3 a 150 . Algumas amostras foram previamente atacadas com HF para eliminação dos silicatos. uma vez que os picos principais da grafita $(3,36 \AA$ e $1,68 \AA$ ) são próximos aos do quartzo (3.34 Á e $1.67 \AA$ ). Tentou-se também identificar a grafita pelo método da pipetagem da suspensão do pó e através de amostras 


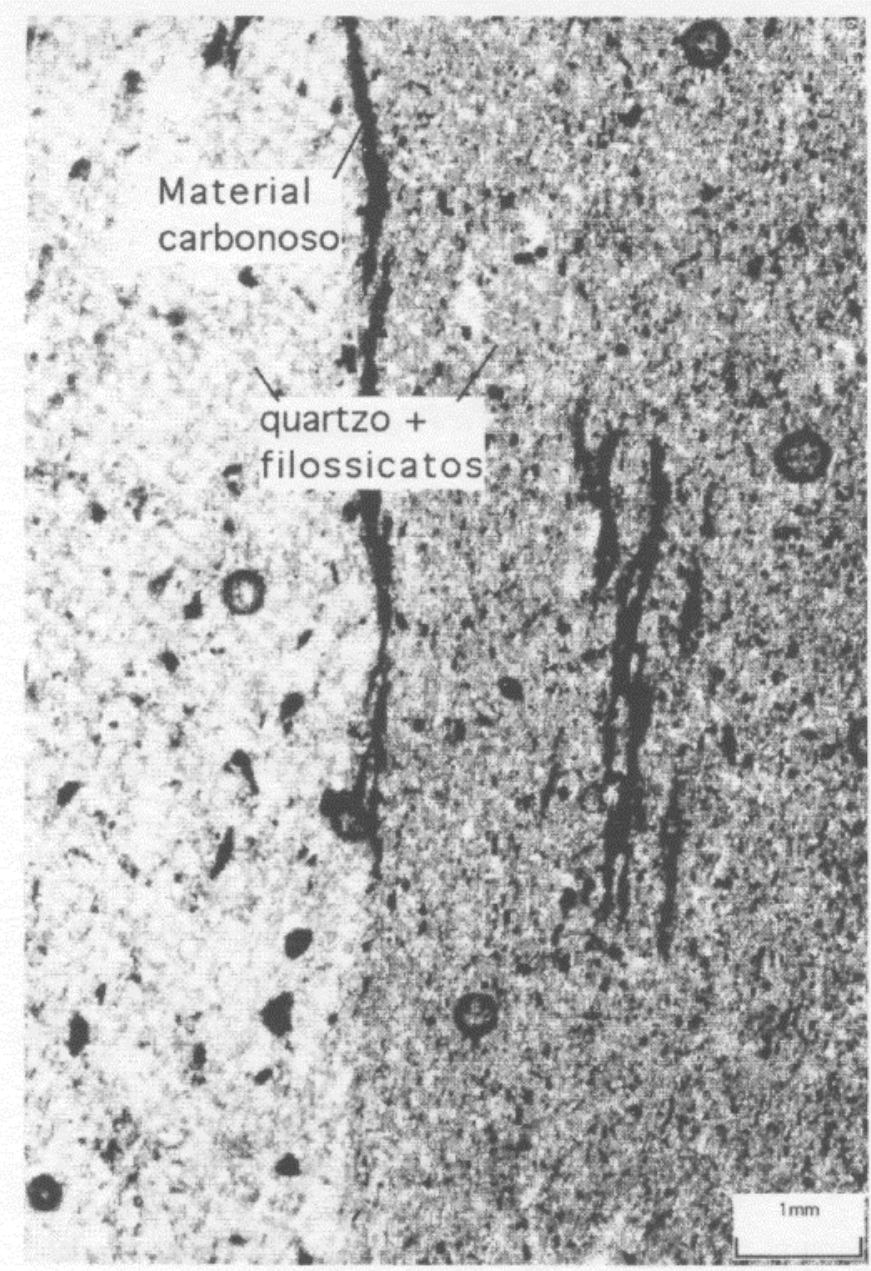

Fotografia 3 - Fotomicrografia defililo carbonoso da Formação Fecho do Funil mostrando material carbonoso disseminado (zonas menos deformados) e reorientados pela deformação. Amostra FF-08-B2. (N//).

Photography 3 - Carbonaceous phylite of the Fecho do Funil Formation showing the behavior of carbonaceous material in deformed and nondeformed zones. Samples FF-08-B2. (Parallel nicols).

orientadas para caracterizar argilominerais, não se obtendo em nenhum caso o espectro da grafita. A maioria das amostras apresentaram linha base com ruídos e irregular, tendo seu ápice próximo a $21^{\circ}$ na posição $2 \Theta$ (Figura 4).

DISCUSSÃO DOS RESULTADOS Os dois principais reservatórios de carbono na crosta terrestre são os de carbono orgânico, com valores de $\delta^{13} \mathrm{C}$ próximos a $-26 \%$ o, e os de carbonato de origem sedimentar, com valores de $\delta^{13} \mathrm{C}$ próximos a 0\%o (Broecker 1970, Schidlowski et al 1975, Veizer et al. 1980). A grande diferença na composição isotópica de carbono desses dois reservatórios deve-se principalmente ao processo de fotossíntese, que favorece significativamente o ${ }^{12} \mathrm{C}$ em detrimento ao ${ }^{13} \mathrm{C}$. As plantas atuais podem ser classificadas de acordo com o processo de fotossíntese que utilizam para fixar carbono em: $\mathrm{C} 3\left(\delta^{13} \mathrm{C}\right.$ $\AA-26 \%), \mathrm{C} 4\left(\delta^{13} \mathrm{C} \hat{\mathrm{A}}-13 \%\right)$ e CAM, com valores intermediários de $\delta^{13} \mathrm{C}$ (Deines 1980).

Baixos valores de $\delta^{13} \mathrm{C}(=-26 \% \mathrm{c})$ em rochas carbonosas arqueanas têm sido considerados como fortes argumentos em favor da existência de fotossíntese desde 3.5 a $3.0 \mathrm{Ga}$ (Schidlowski 1982, Schidlowski et al 1983, Cloud 1983). Os primeiros organismos teriam sido provavelmente bactérias fotossintetizadoras anaeróbicas, que sintetizavam com-
Tabela 1 - Relação das amostras utilizadas nesse trabalho. Table 1 - List of samples used this study.

\begin{tabular}{|c|c|c|c|c|}
\hline Local*Unidade & amostras & C.0.\% & R.I.\% & $\delta^{13} \mathrm{C}$ \\
\hline $\begin{array}{l}\text { 12/Rochas carbonosas } \\
\text { terciárias }\end{array}$ & AM-29 & 24.9 & 70 & -26.9 \\
\hline $\begin{array}{l}\text { 12/Rochas carbonosas } \\
\text { terciárias }\end{array}$ & AM-30 & 29.35 & 73 & -25.7 \\
\hline $\begin{array}{l}\text { 12/Rochas carbonosas } \\
\text { terciárias }\end{array}$ & AM- 45 & 31.92 & 80 & -25.7 \\
\hline $\begin{array}{l}\text { 12/Rochas carbonosas } \\
\text { terciárias }\end{array}$ & AM-50 & 13.73 & 48 & -28.3 \\
\hline $\begin{array}{l}\text { 1/Fecho do Funil. Borra } \\
\text { de café (?) proveniente } \\
\text { de alteração dos carbonatos } \\
\text { da mina do Cumbé. }\end{array}$ & FF-50 & 0.11 & 88 & -23.0 \\
\hline $\begin{array}{l}\text { 8/Olho clínico. Localizado } \\
\text { antes do Ribeirão Gualaxo. }\end{array}$ & OC-1 & 0.64 & 95 & -18.5 \\
\hline 9/Ribeirão Gualaxo. & GL-2 & 0.65 & 95 & -18.7 \\
\hline 9/Ribeirão Gualaxo. & GL-4 & 4.35 & 91 & -21.9 \\
\hline $\begin{array}{l}6 / \text { Trevo Mariana/Ponte } \\
\text { Nova. }\end{array}$ & TR-2 & & & -23.6 \\
\hline $\begin{array}{l}\text { 6/Trevo Mariana/Ponte } \\
\text { Nova. }\end{array}$ & TR-3 & & & -20.6 \\
\hline $\begin{array}{l}\text { 7/Trevo Samitri/Trindade. } \\
\text { Trevo de Capanema. }\end{array}$ & TT-1 & 5.02 & 90 & -29.8 \\
\hline $\begin{array}{l}\text { 4/Mina de Passagem. Filito } \\
\text { carbonoso. }\end{array}$ & PP2-1F & 0.67 & 90 & -17.7 \\
\hline 4/Mina de Passagem. Filito & PP2-1A & 0.74 & 90 & -18.6 \\
\hline $\begin{array}{l}\text { 4/Mina de Passagem. } \\
\text { Quartzito cinza. }\end{array}$ & PP2-1E & 0.12 & 45 & -5.9 \\
\hline $\begin{array}{l}\text { 11/Mina de São Bento. } \\
21.9\end{array}$ & SÃO BENTO & & & - \\
\hline $\begin{array}{l}\text { Itapecirica. Mina Nacional } \\
\text { Grafite. }\end{array}$ & GRAFITA & 40.58 & 90 & -25.8 \\
\hline Mina de Crixás. & CRIXÁS(2) & 1.97 & 90 & -16.2 \\
\hline
\end{tabular}

* - indicado na forma de números, cuja localização encontra-se na figura 1 .

postos orgânicos através da oxidação de compostos de enxofre $\left(\mathrm{H}_{2} \mathrm{~S}\right.$ ou S). Schidlowski (1982) menciona a existência de processos inorgânicos de produção de material carbonoso, tais como Fischer-Tropsch (Lancet \& Anders 1970) e Miller-Urey (Chang et al 1981), que seriam capazes de gerar fracionamento de isótopos de carbono semelhante ao observado no processo de fotossíntese. No entanto, esse mesmo autor argumenta que esses processos são incapazes de fixar quantidades significativas de compostos orgânicos de carbono e de manter o balanço de massa entre os reservatórios de carbono orgânico e inorgânico durante o tempo geológico. Strauss (1986) mostrou que há significativas diferenças entre a composição isotópica de rochas carbonosas arqueanas e proterozóicas. As primeiras apresentam valores de $\delta^{13} \mathrm{C}$ que atingem $-45 \%$ e que têm sido relacionados à atuação de bactérias metanotróficas em ambientes de água estratificada. Baixos valores de $\delta^{13} \mathrm{C}$ também foram descritos em rochas dos Grupos Fartescue, Austrália, Ventersdrop, África do Sul (Hayes et al. 1983), e em rochas do Escudo Canadense (Schoell \& Wellmer 1981). Segundo Strauss (1986), os baixos valores de $\delta^{13} \mathrm{C}$ encontrados em rochas do Arqueano Superior representam uma feição global, tendo sido descritos em quase todos os continentes. De forma geral, os principais fatores que controlam o teor de material carbonoso em rochas são a produção primária de matéria orgânica, a taxa de sedimentação, o grau de oxidação da matéria orgânica na coluna d'água, a degradação pósdeposicional por agentes biológicos, além da diagênese e metamorfismo. 


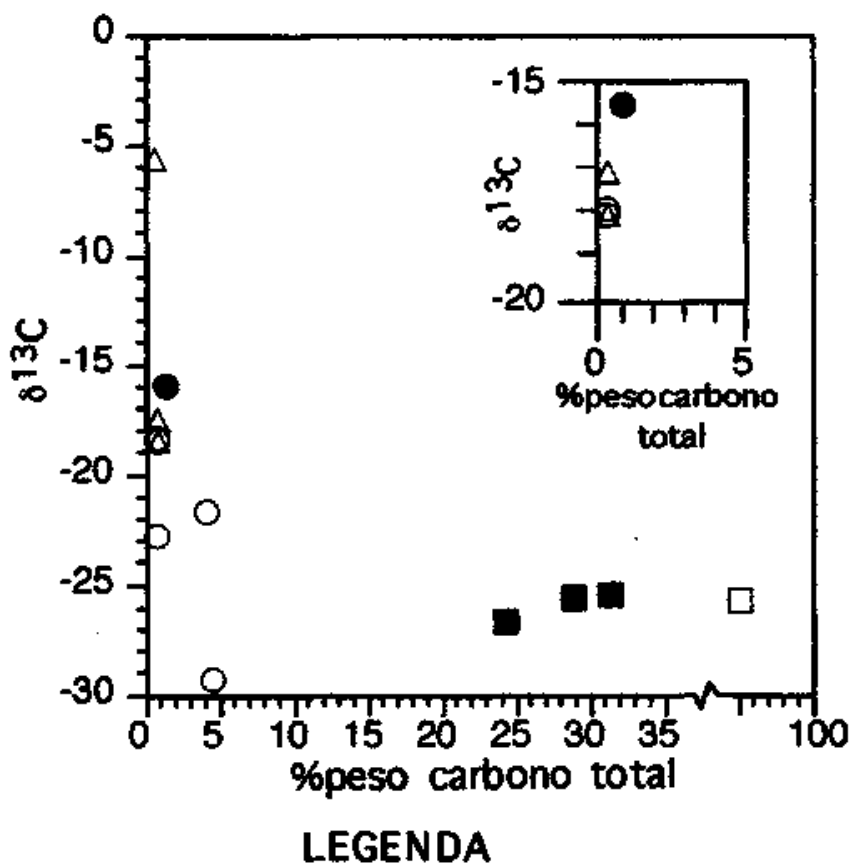

Rochas carbonosas terciárias

O Formagăo Barreiro

\section{$\Delta$ Mina da Passagem}

\section{- Mira de Sâo Bento}

Mina de grafita de Itapecirica

\section{Mira de ouro de Cróxás}

Figura 3 - Distribuição de carbono orgânico total versus $\delta^{19} \mathrm{C}$ de amostras desse estudo.

Figure 3 - Graph displaying the total organic carbon versus the $\delta^{13} \mathrm{C}$ values of the samples.

As amostras deste estudo apresentam valores de $\delta^{13} \mathrm{C}$ compatíveis com carbono de origem orgânica, originados provavelmente através de processo de fotossíntese. No entanto, observam-se diferenças significativas de $\delta^{13} \mathrm{C}$ entre as amostras fanerozóicas e precambrianas, bem como entre as amostras precambrianas. Essas diferenças podem estar relacionadas a processos diagenéticos e metamórficos.

As amostras da Bacia do Gandarela apresentam pequena variação dos valores de $\delta^{13} \mathrm{C}(-28.3$ a $-25.7 \%)$ e teores significativamente maiores de carbono orgânico total (Tabela 1) se comparadas às amostras precambrianas. A pequena variação de $\delta^{13} \mathrm{C}$ indica que essas rochas foram pouco afetadas por processos diagenéticos e pós-diagenéticos. Em geral, esses processos são acompanhados de quebra e liberação de moléculas orgânicas, que tendem a alterar a composição isotópica original do material carbonoso. Com relação às condições paleobotânicas da Bacia do Gandarela, os valores de $\delta^{13} \mathrm{C}$ indicam uma predominância de plantas $\mathrm{C} 3$, provavelmente de origem continental. Essa interpretação está de acordo com dados palinológicos da Bacia do Gandarela, que indicam a existência de plantas fósseis continentais (Lima \& Salard-Cheboldaeff 1981; Pinto \& Pares-Regali 1991).

Os filhos carbonosos precambrianos apresentam, em geral, concentração de carbono orgânico total $<1 \%$ por peso. Esses teores são próximos aos obtidos por Springer (1985) e

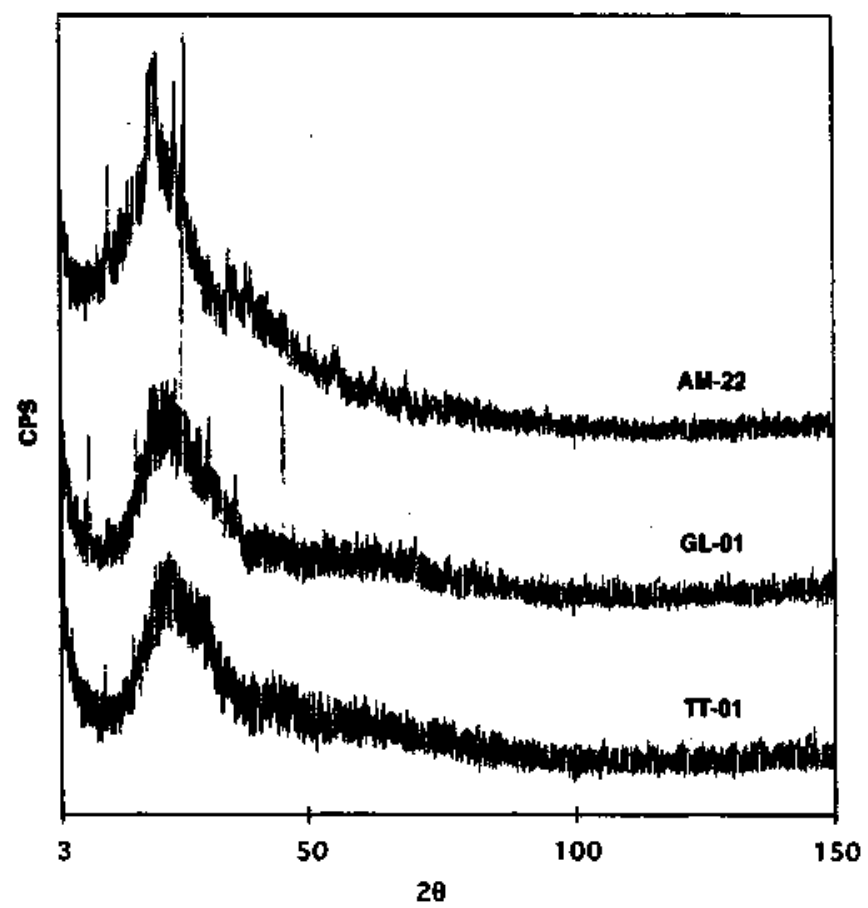

Figura 4 - Espectros de difração de raios-X de material carbonoso de rochas precambrianas (TT-01 e GL-01) comparados com material carbonoso de sedimentos terciários da Bacia do Gandarela (AM-22).

Figure 4 - X-ray patterns of carbonaceous material from Tertiary (AM-22) and Precambrian (TT-01 e GL-01) rocks.

Strauss (1986) para rochas semelhantes do Pré-Cambriano do Escudo Canadense. Os valores de $\delta^{13} \mathrm{C}$ variam entre -29 e $-15 \%$, fazendo-se exceção a uma amostra da mina de Passagem de Mariana, que possui valor de $\delta^{13} \mathrm{C}$ igual a $-5.9 \%$. Essa variação isotópica, mesmo considerando amostras de uma mesma unidade, é reflexo da composição isotópica primária do material carbonoso e de processos sedimentares e metamórficos. Processos metamórficos podem afetar significativamente a composição isotópica de rochas carbonosas, levando à quebra dos compostos orgânicos e liberação de compostos de carbono na forma de $\mathrm{CO}_{2}, \mathrm{CO}, \mathrm{CH}_{4}$, dentre outros (Hoefs \& Frey 1976). Estudos experimentais mostram que durante o processo de degradação térmica de compostos orgânicos as ligações ${ }^{12} \mathrm{C}-{ }^{\mathrm{I}} \mathrm{C}$ tendem a romper-se mais facilmente que ligações do tipo ${ }^{12} \mathrm{C}-{ }^{13} \mathrm{C}$, levando a um enriquecimento $\mathrm{em}^{13} \mathrm{C}$ no material residual se comparado aos compostos volatilizados. A variação de $\delta^{13} \mathrm{C}$ nessas rochas indica também de que o processo metamórfico ocorreu em sistema aberto, pelo menos em termos de compostos de carbono. Essa discusão é relevante, levando-se em consideração que amostras de grafita associadas a rochas de alto grau metamórfico, tais como as de Itapecirica, MG, apresentam valores de $\delta^{13} \mathrm{C}(-25 \%)$, ou seja, próximo a valores de materiais carbonosos não alterados. Isso indica que o metamorfismo em Itapecirica ocorreu provavelmente em sistema fechado ou sem a perda significativa de compostos de carbono. Como discutido por Weis et al. (1981), rochas carbonosas submetidas a metamorfismo de alto grau não são acompanhadas, aparentemente, por perda e circulação de hidrocarbonetos resultantes da decomposição da matéria orgânica. O mesmo, no entanto, não se observa nas amostras de material carbonoso do Quadrilátero Ferrifero, que apresentam variações significativas de $\delta^{\prime 3} \mathrm{C}$, sugerindo que durante $\mathrm{o}$ processo metamórfico, esses filhos carbonosos podem ter perdido carbono na forma de $\mathrm{CO}, \mathrm{CO}_{2}$ e $\mathrm{CH}_{4}$. Muito embora 
não se saiba as condições em que essa volatilização ocorreu, sugere-se que a quebra desses compostos orgânicos tenha sido uma possível fonte de voláteis, tais como $\mathrm{CO}_{2} \mathrm{e} \mathrm{CH}_{4}$, durante o metamorfismo.

CONCLUSÕES As amostras de filitos carbonosos do Quadrilátero Ferrifero estudadas nesse trabalho não apresentam estrutura cristalina organizada, não podendo ser classificadas como grafita. $\mathrm{O}$ material carbonoso possui estrutura semi-organizada e apresenta em termos de difração de raios-X um pico principal significativamente mais largo que o da grafita de alta temperatura.

Os dados de isótopos estáveis indicam que o material carbonoso apresenta, via de regra, valores de $\mathrm{d}^{13} \mathrm{C}$ abaixo de $-15 \%$, indicando uma provável origem orgânica. Observa-se também que o material carbonoso de rochas precambrianas possui uma variação significativa $(-29.8$ a $-18.6 \%$ ), que está relacionada provavelmente a processos pós-sedi- mentares e metamórficos. Esses processos tendem a quebrar a matéria orgânica e liberar compostos de carbono, tais como $\mathrm{CO}_{2}$ e $\mathrm{CH}_{4}$. Sugere-se ainda que o metamorfismo dessas rochas ocorreu em sistema aberto e que a liberação desses voláteis tenha sido uma possível fonte de fluidos metamórficos no Quadrilátero Ferrifero. Em contraste com as amostras precambrianas, as amostras terciárias apresentam uma menor variação de $\mathrm{d}^{13} \mathrm{C}$ indicando que foram pouco afetadas por processos pós-sedimentares.

Agradecimentos Os autores agradecem Fernando de M. Krahenburhl e Ricardo Perobelli Borba durante os trabalhos de campo, e ao Prof. Newton Souza Gomes pela revisão e discussão do texto. Agradecem também ao Departamento de Metalurgia da UFOP e à Petrobrás, pelas análises de carbono total, e ao CNPq (Proc.40.4814/87-5) pelo apoio financeiro.

\section{REFERÊNCIAS BIBLIOGRÁFICAS}

BROECKER W.S. 1970. A boundary condition on the evolution at atmospheric oxygen. J. Geophys. Res., 75:3553-3557.

BUSECK P.R. \& BOJUN H. 1985. Conversion of carbonaceous materia to graphite during metamorphism. Geochim. Cosmochim. Ac/a, 49(10):2003-2016

CHANG S.; DBS MARAIS D; MACK R.; MILLER S.L. \& STRATHEARN G. 1981. Prebiotic organic syntheses and the origin of life. In: SCHOPF, J.W. ed.Origin and Evolution of Earth's Earliest Biosphere, Princenton, NJ. Princeton University Press, p. 53-92.

CHEMALE JR. FARID; ROSIERE C.A. \& ENDO I. 1994. The tectonic evolution of the Quadrilátero Ferrifero, Minas Gerais, Brazil. Precam. Res., 65:25-54.

CLOUD P. 1983. Early biogenic history; the emergence paradigm. In SCHOPF, J.W. ed.Earths's Earliest Biosphere; Its Origin and Evolution, Princenton, NJ, Princenton Univ. Press., p. 14-31.

DEINES P. 1980. The isotopic composition of reduced organic carbon. In: FRITZ, H. \& FONTES J.CH., eds. Handbook of Environmetal Isotope Geochemistry, Elsevier, Amsterdam, vol. 1 A, pp. 320-406

DERRY L.A.; KAUFMAN AJ. \& JACOBSEN S.B. 1992. Sedimentary cycling and environmental change in the Late Proterozoic: Evidence from stable and radiogenic isotopes. Geoch. Cosmoch. Acta, 56:1317-1329.

DES MARAIS D.J.; STRAUSS H.; SUMMONS R. \& HAYES J.M. 1992 Carbon isotope evidence for the stepwise oxidation of the Proterozoic environment. Nature, 359:605-609.

DUARTE L. \& MELLO P M. DA C. 1980. Flórula Cenozóica de Gandarela, MG, I. An. Acad. Brás. Ciênc., 52(1):77-91.

FAURE G. 1986. Principles of Isotope Geology. John Wiley \& Sons, 2nd ed., 589 pps.

GORCEIX, H. 1884. Bacias terciárias d'agua doce nos arredores de Ouro Preto (Gandarela e Fonseca., Minas Gerais, Brasil. An. Escola de Minas de Ouro Preto, 3:75-92.

GRIFFIN, G.M. 1967. X-ray diffraction techniques applicable to study of diagenesis and low rank metamorphism in humic sediments. /. Sed. Petrol, 37:1006-1011.

GUHA J.; TAIT L.; HEROUX Y. \& GARNEAU F.-X. 1984. Character and partitioning of elements in organic matter within black shales preliminary findings. Geol. Assoc. of Can./ Min. Assoc. Can. Programs with abstracts, p. 69

HAYES J.M.; KAPLAN I.R. \& WEDEKING K.W. 1983. Precambrian organic geochemistry: Preservation of the record. In: SCHOPF, J.W. ed.Earths's Earliest Biophere; Its Origin and Evolution, Princenton, NJ, Princenton Univ. Press, p. 95-135.

HOEFS J. \& FREY M. 1976. The isotopic composition of carbonaceous matter in a metamorphic profile from the Swiss Alps. Geochim. Cosmochim. Acta, 40:945-951.

HOEFS J. 1980. Stable Isotope Geochemistry. 2nd ed., Springer Verlag, New York, 208pp.

LADEIRA E.A. 1980. Metallpgenesis of Gold at the Morro Velho Mine, and in Nova Lima District, Quadrilátero Ferrifero, Minas Gerais, Brazil. Tese de Doutorado, University of West Ontario, London, $272 \mathrm{pp}$.
LANCET D.R \& ANDERS E. 1970. Carbon isotope fractionation in the Fisher-Tropsch synthesis and in meteorites. Science, 170:980-982.

LIMA M.R. \& SALARD-CHEBOLDAEFF M. 1981. Palynologie des bassins de Ganderela et Fonseca (Eocenede L'Etat de Minas Gerais, Brésil). Boletim, Instituto de Geociências, USP, 12:33-54.

KAUFMAN A.J.; JACOBSEN S.B. \& KNOLL A.H. 1993. The Vendian record of $\mathrm{Sr}$ and $\mathrm{C}$ isotopic variations in seawater: Implications for tectonics and paleoclimate. Earth Planet. Sci. Lett., 120:409-430.

MAGARITZ M.; HOLSER W.T. \& KIRSCHVINK J.L. 1986. Carbonisotope events across the Precambrian/Cambrian boundary on the Siberian Platform. Nature, 320:258-259.

PINTO A.D.P \& PARES-REGALI, M.S. 1991. Palinoestratigrafia dos sedimentos terciários da bacia de Gandarela, Minas Gerais, Brasil. Rev. Escola de Minas, 44(1): 10-15.

SCHIDLOWSKI M. 1982 Content and isotopic composition of reduced carbon in sediments. In: HOLLAND H.D. \& SCHIDLOWSKI M. eds Mineral Deposits and the Evolution of the Biosphere, Berlin, Holland \& Springer Verlag, 103-122.

SCHIDLOWSKI M.; EICHMANN R. \& JUNGE CH.E. 1975. Precambrian sedimentary carbonates; carbon and oxygen isotope geochemistry and implications for the terrestrial oxygen budget. Precambrian Res., 2:1-69.

SCHIDLOWSKI M.H.; HAYES J.M. \& KAPLAN E.R. 1983. Isotopic inferences of ancient biochemistries: carbon, sulfur, hydrogen, and nitrogen. In: SCHOPF, J.W. ed. Earths's Earliest Biophere; Its Origin and Evolution, Princenton, NJ, Princenton Univ. Press.., p. 149-186.

SCHOEL M. \& WELLMER F.-W. 1981. Anomalous $\delta^{13} \mathrm{C}$ depletion in early Precambrian graphites from Superior Province, Canada. Nature, 290:696-699.

SPRINGER J. S. 1985. Carbon in Archean rocks of the Abitibi belt (Ontario-Quebec) and its relation to gold distribution. Can. J. Earth Sci.. 22:1945-1951.

STARLING A.; GILLIGAN J.M.; CARTER A.H.J.; FOSTER R.P. \& SAUNDERS R.A. 1989. High-temperature hydrothermal precipitation of precious metals on the surface of pyrite. Nature, 340:298-300.

STRAUSS H. 1986. Carbon and sulfur isotopes in Precambrian sediments fom the Canadian Shield. Geochim. Cosmochim. Acta, 50:2653-2662.

VEIZER J.; HOLSER W.T. \& WILGUS C.K. 1980. Correlation of $\delta^{13} \mathrm{C} / 12 \mathrm{C}$ and $34 \mathrm{~S} / 32 \mathrm{~S}$ secular variations. Geochim. Cosmochim. Acta. 44:579-587.

VOLKOV I.I. \& FOMINA L.S. 1974. Influence of organic material and processes of sulphide formation on distribuition of some trace elements. In: DEGENS E.T. E ROSS D.A. eds.The Black Sea-Geology, Chemistry and Biology, Assoc. Am. Petro. Geol., Memoir 20:456-477.

WEIS P. L.; FRIEDMAN I. \& GLEASON J.P. 1981. The origin of epigenetic graphite: evidence from isotopes. Geochim. Cosmochim. Acta, 45:2325-2332.

MANUSCRITO A838 Recebido em 22 de agosto de 1995 Revisão do autor em 12 de dezembro de 1995 Revisão aceita em 20 de dezembro de 1995 